\title{
Acretismo placentário do tipo placenta percreta: um relato de caso
}

\section{Placental acretism of the type percrete placenta: a case report}

\author{
Vanessa Pimentel Leal, ${ }^{1}$ Graciete Helena Nascimento dos Santos, ${ }^{2}$ \\ Lilalea Goncalves França ${ }^{3}$
}

RESUMO: Esse estudo teve como objetivo descrever um relato de caso clínico com uma revisão de literatura sobre acretismo placentário do tipo placenta percreta, que é uma condição considerada rara e que apresenta taxas de mortalidade e morbidade relativamente altas para a mãe e o feto. A placenta percreta é classificada como um tipo de placenta que foi aderida de forma anormal, em que as vilosidades coriônicas acabam fortalecendo-se e aderindo através da parede uterina em toda a sua extensão, podendo inclusive crescer em órgãos da cavidade abdominal. Dentre os tipos de acretismo placentário, a placenta percreta é a forma mais invasiva no que diz respeito à anormalidade da implantação placentária, em que o tecido das vilosidades coriônicas invadem a total espessura uterina através da serosa. Dessa forma, este artigo visa relatar um caso de acretismo placentário do tipo placenta percreta, que não foi diagnosticado por ultrassonografia em idade gestacional de aproximadamente 32 semanas e 3 dias, sendo a paciente submetida à cesariana de emergência devido a quadro sintomático. Durante o procedimento cirúrgico, foi constatada uma rotura uterina em região segmentar do útero de forma ascendente com extravasamento de feto, placenta e sangramento ativo em cavidade abdominal.

PALAVRAS CHAVE: placenta percreta; acretismo placentário; rotura uterina.

ABSTRACT: This study aimed to describe a clinical case report with a review of the literature on placental acretism the percrete placenta type, which is a condition considered rare and presents relatively high mortality and morbidity rates for the mother and the fetus. The peritoneal placenta is classified as a type of abnormally adherent placenta, in which the chorionic villi strengthen and adhere through the uterine wall in all its extension, and may even grow in organs of the abdominal cavity. Among the types of placental accretion, the peritoneal placenta is the most invasive form with respect to the placental implantation abnormality, in which the tissue of the chorionic villi invades the total uterine thickness through the serosa. Thus, this article aims to report a case of peritoneal placenta accreta, which was not diagnosed by ultrasonography at gestational age of approximately 32 weeks and 3 days, and the patient underwent emergency cesarean section due to symptomatic symptoms. During the surgical procedure, uterine rupture was observed in the uterine segment of the uterus in an ascending way with extravasation of the fetus, placenta and active bleeding in the abdominal cavity.

KEYWORDS: placenta percreta; placental acretism; uterine rupture.

1. Médica e egressa da UNICEUMA. Residente em Ginecologia e Obstetrícia pelo Hospital Universitário da Universidade Federal do Maranhão. E-mail: vanessa_pleal@ hotmail.com

2. Doutora em Saúde Coletiva - UFMA. Mestra em Ciências da Saúde - UFMA. Médica Ginecologista e Obstetra. Preceptora da Residência Médica em Ginecologia e Obstetrícia e chefe da Unidade de Atenção a Saúde da Mulher - Hospital Universitário da Universidade Federal do Maranhão. E-mail: gracietehelenans@yahoo.com.br

3.Mestra em Ciências Biológicas (Biofísica) pela UFRJ em convenio com a University of Maryland at Baltimore-USA. Doutoranda em Biotecnologia (Renorbio) - UFMA. Docente colaboradora da UNICEUMA e assistente IV da UFMA. Pesquisadora coordenadora nos projetos de pesquisa: Avaliação da ação analgésica e antioxidante de Persea americana Mill. em ciatalgia de roedores - Financiadora: FAPEMA, Levantamento sobre o uso de drogas psicoativas entre os estudantes dos cursos de Medicina e Enfermagem de uma universidade particular em São Luís - MA - Financiadora: UNICEUMA, Atividade de plantas da flora maranhense e sintéticos bioativos em modelo experimental de dor neuropática - FAPEMA, Sistema único de saúde: avaliação da qualidade segundo a percepção de usuários e médicos e Monitoração da atividade espasmolítica de produtos obtidos de arrabidaea chica (hbk) verlot. E-mail: lilafranca@gmail.com 


\section{Introdução}

Acretismo placentário do tipo placenta percreta é uma condição considerada rara e que apresenta taxas de mortalidade e morbidade relativamente altas para a mãe e o feto. O manejo e diagnóstico precoce são difíceis sendo que a literatura descreve poucos relatos de diagnóstico ultrassonográfico acurado durante o segundo trimestre gestacional (HUBINONT et al., 2017; JAUNIAUX; COLLINS; BURTON, 2017).

A placenta percreta, é classificada como um tipo de placenta que foi aderida de forma anormal, em que as vilosidades coriônicas acabam fortalecendo-se e aderindo através da parede uterina em toda a sua extensão, podendo inclusive crescer em órgãos da cavidade abdominal. A sua incidência aumenta de forma proporcional ao número de cesáreas anteriores, outros procedimentos cirúrgicos que foram realizados no útero, como também a idade materna e o uso de nicotina (HUBINONT et al., 2017; ROLNÁ; MATLÁK; DVOŘÁČKOVÁ, 2017).

Dessa forma, esse artigo visa relatar um caso de acretismo placentário do tipo placenta percreta, que não foi diagnosticado por ultrassonografia em idade gestacional de aproximadamente 32 semanas e 3 dias, sendo a paciente submetida à cesariana de emergência devido a quadro sintomático. Durante o procedimento cirúrgico, foi constatada uma rotura uterina em região segmentar do útero de forma ascendente com extravasamento de feto, placenta e sangramento ativo em cavidade abdominal.

\section{Material e Métodos}

Trata-se de um estudo de caso clínico com abordagem descritiva básica com revisão de literatura, o estudo foi realizado com uma única amostra e visou apresentar o relato de caso clínico e cirúrgico, a fim de elucidar a comunidade acadêmica da área da saúde, bem como os profissionais médicos, em relação aos casos de rotura uterina por acretismo placentário do tipo placenta percreta e sua incidência, sendo analisados artigos já publicados sobre acretismo placentário do tipo placenta percreta.

A estratégia de busca eletrônica teve os seguintes descritores: "placenta percreta", "acretismo placentário", "rotura uterina", e suas correspondentes em inglês "placenta percreta", "placental acretism", "uterine rupture". Foram incluídas publicações em língua portuguesa e inglesa.

Os artigos foram pré-selecionados a partir da leitura do resumo disponível nas bases de dados da SCIELO, PUBMED e BMJ, dos artigos publicados no período de 2008 a 2018. O estudo 
foi aprovado pelo Comitê de Ética em Pesquisa do Hospital Universitário da Universidade Federal do Maranhão ( $\mathrm{N}^{\mathrm{o}}$. do parecer consubstanciado: 2.525.674).

\section{Descrição do Caso e discussão}

LOS, 26 anos, feminino, Gesta: II Para: 0 Aborto: I, idade gestacional (IG): 32 semanas e 3 dias, parda, natural e residente em Santa Luzia do Parua - MA, religião não informada. Foi admitida ao Pronto Socorro Obstétrico, referindo quadro álgico pélvico e perineal com perda de líquido.

Ao exame físico, pressão arterial (PA): 120x70 milímetros de mercúrio (mmHg), frequência cardíaca (FC): 76 batimentos por minuto (bpm), saturação de oxigênio: 99\%, lúcida, orientada em tempo e espaço, dinâmica uterina ausente, tônus uterino normal, batimento cardíaco fetal (BCF): 145 bpm, toque vaginal apresenta colo grosso, fechado, sem perdas e não foi observado perda de líquido a manobra de valsalva. Como conduta inicial foi instituída analgesia e solicitado Ultrassonografia (USG) obstétrica de emergência.

A USG evidenciou IG: 32 semanas e 3 dias, feto único, vivo, cefálico, BCF: 149 bpm, movimento fetal $(\mathrm{MF})+$, peso de $1.993 \mathrm{~g}$, placenta anterior ao seguimento inferior do útero, índice do líquido amniótico: 60mm, diante dos achados foi solicitado internação da paciente em enfermaria gestacional de alto risco por suspeita de Rotura Prematura de Membranas Ovulares (RPMO).

No 00 dia de internação hospitalar (DIH), foi instituído cuidados gerais e inicio da administração de dexametasona. Ao final do dia a paciente evoluiu com dor em região pélvica sendo administrado à terceira dose de dexametasona e controle da vitalidade fetal.

No $01^{\circ} \mathrm{DIH}$, a paciente evoluiu com mal estar geral, astenia, obnubilação, dor pélvica intensa e hipotensão súbita (60 x $30 \mathrm{mmHg})$, ao exame físico apresentava-se em ruim estado geral, hipocorada (4+/4+), consciente, desorientada, tônus uterino de difícil avaliação devido a quadro álgico notando-se tônus aumentado à esquerda e amolecido a direita, dinâmica uterina ausente, BCF: 90 bpm. Mediante avaliação clínica foi instituída como conduta hidratação vigorosa e indicação de cesárea de urgência por bradicardia fetal.

Procedimento cirúrgico realizado sob anestesia geral, incisão da pele em laparotomia mediana infraumbilical e abertura da cavidade abdominal por planos anatômicos, realizado inventário da cavidade abdominal, visualizando feto e placenta livres na cavidade, grande quantidade de sangue vivo e com coágulos em abdome, procedeu-se à retirada do feto (em morte 
aparente, depois constatado pela equipe pediátrica), seguida da retirada da placenta e limpeza da cavidade abdominal; foi necessária a transfusão de 02 concentrados de hemácias no intra operatório devido a choque hipovolêmico no transoperatório, o útero de aproximadamente $30 \mathrm{~cm}$ de diâmetro, encontrava-se amolecido com rotura uterina em região segmentar em formato oblíquo ascendente. $\mathrm{Na}$ figura 1 foi constatado acretismo placentário do tipo placenta percreta e anexos sem anormalidades bilateral, dessa forma optou-se por realizar conduta conservadora após acordo conjunto com a equipe obstétrica, procedendo-se a limpeza da cavidade uterina, escarificação das bordas da rotura com intuito da revitalização do epitélio uterino, histerorrafia verificando-se uma discreta atonia em que foi administrado ocitocina intra cornual, seguindo com a limpeza da cavidade abdominal e lavagem com soro fisiológico $0,9 \%$ até saída de líquido claro.

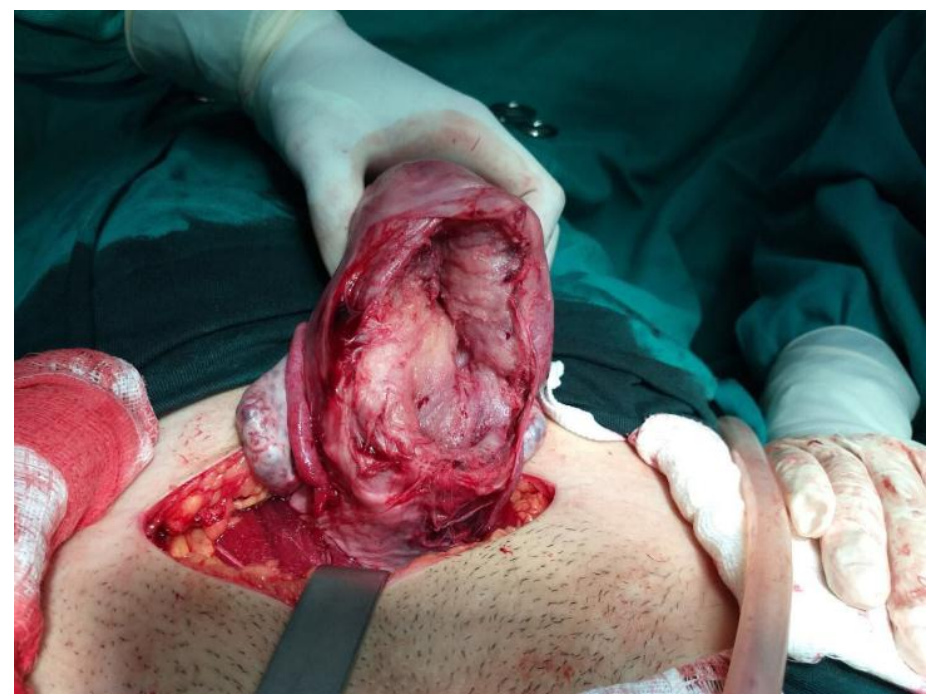

Figura 1. Rotura uterina em região segmentar em formato oblíquo ascendente.

Seguiu-se pela revisão da cavidade abdominal e da hemostasia, constatou-se útero contraído e seguiu-se com fechamento da cavidade dando início pela aproximação do peritônio parietal com sutura contínua simples (fio vicryl 2.0), aproximação da musculatura com sutura simples (fio vicryl 2.0), fechamento da aponeurose com sutura contínua ancorada (fio vicryl 0), sutura simples de pele (fio nylon 4.0). Ao fim do procedimento, procedeu-se à limpeza e colocação de curativo compressivo, sendo a placenta encaminhada ao anátomo-patológico.

O diagnóstico pós-cirúrgico foi de rotura uterina por acretismo placentário do tipo placenta percreta. Paciente deixou a sala de cirurgia em regular estado geral, sendo então admitida à unidade de terapia intensiva (UTI), sonolenta, respondendo a solicitações verbais, hipocorada, em infusão de concentrado de hemácia, respiração espontânea com cateter de oxigênio nasal, normocárdica, ao exame físico: PA: 114x64 mmHg, FC: 88 bpm, frequência respiratória (FR): 20 incursões respiratórias por minuto (IRPM), temperatura axilar: $36^{\circ} \mathrm{C}$, glicemia capilar: $132 \mathrm{mg} / \mathrm{dL}$, aparelho 
respiratório: murmúrios vesicular positivo, bilateralmente e crepitações em base direita, aparelho cardiovascular: bulhas normofonéticas em 2 tempos sem sopros, abdome: globoso, distendido, ruídos hidroaéreos débil, útero em involução, extremidades com edema: 3+/4+, pulsos presentes, aparelho neurológico: escala de coma de glasgow: 15, pupilas isocóricas e fotorreativas. Como conduta, foram solicitados exames laboratoriais de rotina e antibiótico terapia com ceftriaxona e metronidazol.

No $03^{\circ} \mathrm{DIH}$ e $01^{\circ}$ dia de pós-operatório (DPO), paciente apresentava-se em grave estado geral, respiração confortável em ar ambiente, consciente e orientada, hemodinamicamente estável, sem uso de droga vasoativa, normotensa, normocorada, diurese presente $300 \mathrm{ml} / 4 \mathrm{~h}$, afebril, dor difusa em abdome na palpação,

No $04^{\circ} \mathrm{DIH}$ e $02^{\circ} \mathrm{DPO}$, paciente apresentou-se com melhora do quadro e passou para dieta oral, seguindo sem intercorrências. Foi submetida à USG abdominal que evidenciou órgãos ecograficamente normais e exames laboratoriais demonstraram melhora do quadro de infecção.

No $05^{\circ} \mathrm{DIH}$ e $03^{\circ} \mathrm{DPO}$, paciente estável em boas condições hemodinâmicas, com diurese espontânea e satisfatória (2.175 mililitros $(\mathrm{ml}) / 24$ horas $(\mathrm{h}))$, sem febre, ferida operatória (FO) em bom aspecto de cicatrização, boa aceitação da dieta oral e como conduta foi instituída alta da UTI.

Paciente evoluiu assintomática em internação na enfermaria, fazendo uso de antibioticoterapia, com FO limpa sem sinais de infecção, afebril, com mamas não lactantes, com útero contraído e liberação de lóquios fisiológicos, recebeu alta hospitalar no $12^{\circ} \mathrm{DIH}$ e $10^{\circ} \mathrm{DPO}$ sem qualquer queixa.

O laudo histopatológico da placenta evidenciou vilosidades coriônicas apresentando hiperplasia de nós sinciciais e deposição de fibrina intra e intervilositária. Membranas corioamióticas sem alterações histológicas significativas e cordão umbilical sem alterações.

Dentre os tipos de acretismo placentário, a placenta percreta é a forma mais invasiva no que diz respeito à anormalidade da implantação placentária, em que o tecido das vilosidades coriônicas invadem a total espessura uterina através da serosa (HUBINONT et al., 2017; JAUNIAUX; COLLINS; BURTON, 2017). A literatura registra que, em muitos casos de placenta percreta, poderá ocorrer uma situação em que o tecido viloso invade os tecidos e órgãos pélvicos circundantes, ao útero como a bexiga (HUBINONT et al., 2017). Sendo que a possibilidade das vilosidades coriônicas em agregar-se aos órgãos circundantes leva ao comprometimento da sua integridade (seja do trato gastrointestinal ou da própria bexiga urinária) e pode acarretar fezes com 
sangue, hematúria ou complicações urológicas mais graves (ROLNÁ; MATLÁK; DVOŘÁČKOVÁ, 2017).

O primeiro caso de placenta percreta descrito na literatura foi realizado por Alexandroff no ano de 1900, sendo que registros apontam que até o ano de 1956, apenas oito casos tinham sido relatados de placenta percreta associada à rotura uterina, sendo que dessas apenas uma apresentava cesariana prévia (HUBINONT et al., 2017).

Um estudo que avaliou a prevalência do acretismo placentário, apontou que aproximadamente $25 \%$ dos 203 casos incluídos no mesmo foram confirmados com diagnóstico de placenta percreta. Dessa forma, esses dados revelam que a placenta percreta é a condição de maior complexidade, estando associada a uma alta taxa de morbidade materna (JAUNIAUX; BHIDE, 2017).

Outro estudo demonstrou que até meados de 1960, a maioria dos transtornos associados ao acretismo placentário esteve associado à endometrite, remoção manual da placenta e também a curetagem uterina. Entretanto, após esse período constata-se que a maioria dos casos relacionados a esse distúrbio, está relacionada a mulheres que apresentam cicatriz anterior de cesariana (HUBINONT et al., 2017). Com isso, outros fatores de risco significativos podem também ser incluídos como procedimentos cirúrgicos uterino, idade materna ou abuso de nicotina (ROLNÁ; MATLÁK; DVOŘÁČKOVÁ, 2017; SUN et al., 2018; TANTBIROJN; CRUM; PARAST, 2008).

Essa condição se justifica, pois os defeitos que são encontrados nas cicatrizes de cesarianas anteriores apresenta uma degeneração miometrial, bem como uma reduzida ou até mesmo ausente reepitelização na local da cicatriz. E atualmente é sabido que as cicatrizes uterinas apresentam impacto direto na implantação placentária (HUBINONT et al., 2017; JAUNIAUX; COLLINS; BURTON, 2017).

Dados epidemiológicos demonstraram um aumento de $50 \%$ do risco de desenvolver placenta prévia se a paciente apresentou um parto cesariano anterior, e caso tenho passado por dois partos anteriores de cesariana esse risco aumente para $100 \%$. Um relevante estudo apontou que cerca de 59\% das mulheres que apresentaram placenta percreta evoluíram com complicações intra e pós-operatórias, como hemorragias, lesão de bexiga, embolia pulmonar e parada cardiorrespiratória (PCR) (HUBINONT et al., 2017; LEVIN et al., 2017).

Evidências crescentes apontam que as mulheres com placenta percreta, muitas vezes fazem jus à transfusão de derivados sanguíneos como também necessitam de cuidados da UTI, além de 
apresentarem maior incidência de complicações urológicas, como lesão de ureteres. Justifica-se assim, cada vez mais, a necessidade de um atendimento especializado e que disponha de equipe multidisciplinar para seguimento e acompanhamento do desfecho clínico (HUBINONT et al., 2017; LEVIN et al., 2017; ROLNÁ; MATLÁK; DVOŘÁČKOVÁ, 2017).

Via de regra os transtornos placentários apresentam-se na maioria das vezes de maneira assintomática até o parto. Entretanto, é comum por se apresentarem com sangramentos significativos e com risco iminente à vida (ROLNÁ; MATLÁK; DVǑ̌ÁČKOVÁ, 2017).

O diagnóstico dos distúrbios placentários é complicado, e muitas vezes só é possível de ser caracterizado perioperatoriamente. Dessa forma, o exame de ultrassonografia e a ressonância magnética estão entre os métodos diagnósticos mais implementados. A partir da ultrassonografia é possível verificar as lacunas vasculares atípicas, diminuição da espessura do miométrio, perda da linha de hipoecogenicidade, ruptura da linha vesico uterina e ainda o fluxo de alta velocidade dos vasos sanguíneos retroplacentários. Já com o uso da ressonância magnética, podemos obter informações quanto à localização da placenta ou até mesmo dados sobre a invasão dos órgãos circundantes (LEVIN et al., 2017; ROLNÁ; MATLÁK; DVOŘÁČKOVÁ, 2017).

Cabe citar que a sensibilidade do exame é dependente de determinadas nuances como o operador, a idade gestacional em que a paciente se encontra e local de implantação placentária (LEVIN et al., 2017; ROLNÁ; MATLÁK; DVOŘÁČKOVÁ, 2017).

Durante o parto, em geral é que ocorre o diagnóstico dessa condição e assim devem ser tomadas medidas importantes nesse momento, que muitas vezes indicam uma histerectomia. Alguns estudos apontam para uma tendência de um tratamento mais conservador, visando que o útero seja poupado, principalmente quando a mãe é jovem, que apresenta desejo de gravidez futura e não possui proles, entretanto é uma medida que deve ser muito bem planejada pela equipe, por apresentar várias complicações além de altas taxas de morbidade e mortalidade, assim o tratamento conservador requer indicações precisas e um seguimento rigoroso (HUBINONT et al., 2017; ROLNÁ; MATLÁK; DVOŘÁČKOVÁ, 2017).

A tempo, um estudo relatou com sucesso o uso combinado de ácido tranexâmico e enoxaparina para o manejo conservador de uma paciente que começou a desenvolver um quadro de coagulação intravascular disseminada, resultante da placenta percreta. Com isso, vale citar que para um manejo conservador da placenta percreta com segurança, é importante evitar a separação parcial iatrogênica da placenta (MATSUZAKI et al., 2017). 
Os dados epidemiológicos demonstram que embora muitas vezes optou-se por um manejo conservador inicial, aproximadamente $40 \%$ das mulheres com placenta percreta acabam sendo submetidas à histerectomia de emergência e que $42 \%$ apresentam maior morbidade. Dessa forma, ressalte-se que é necessária uma observação cuidadosa para o manejo conservador da placenta percreta, bem como uma assistência adequada na recuperação que visa um cuidado intensivo acurado (MATSUZAKI et al., 2017).

Vários estudos de placentas de gestantes em pré-eclâmpsia e hipertensas apresentam alterações em sua macro e microspia no que diz respeito a áreas de infarto e hematomas, e também relatam o acúmulo de fibrina na túnica íntima dos vasos, o que pode levar a oclusão do mesmo. Via de regra em nível microscópico esses infartos apresentam-se histologicamente com áreas de tecido vilositário necrótico. Em geral esses tecidos podem ser comprimidos pelos hematomas e assim gerar infartos e hipóxia. Fisiopatologicamente o descolamento prematuro da placenta, pode ocorrer nessas áreas de infartos (ARTICO et al., 2017).

Estudos de microscopia eletrônica têm-se especializado em descrever nós sinciciais como células envelhecidas, que estão agrupadas sob a forma de nós imersos nas vilosidades coriônicas. Em gestações normais até a $20^{\mathrm{a}}$ semana, muito raramente são encontrados nós sinciciais, visto que, com o avançar gestacional, os mesmos passam a aumentar sua frequência (ARTICO et al., 2017).

Em geral, sua frequência é bastante aumentada em placentas oriundas de gravidez complicada. Em um estudo autores propuseram que os nós sinciciais estavam localizados em quase todas as vilosidades terminais das placentas de pacientes que sofreram de pré-eclâmpsia, sendo que em seus estudos constataram a prevalência dos mesmos em cerca e $15 \%$ das placentas normais (NARASIMHA; VASUDEVA, 2011). Dessa forma o aumento dos nós sinciciais passaram a ser atribuídos a uma condição preditora ao envelhecimento precoce da placenta, ao passo que coincide a fisiopatologia da pré-eclâmpsia (ARTICO et al., 2017).

Por fim, o aumento da prevalência dos nós sinciciais agregados à necrose das vilosidades coriônicas e o depósito de fibrina intervilositária tendem a possível constatação de uma síndrome hipertensiva devido ao processo de envelhecimento da placenta (ARTICO et al., 2017).

\section{Conclusões}

O trabalho permite concluir que essa patologia aumenta consideravelmente com o histórico de incisões por parto cesariana, com o aumento da idade materna, o uso de nicotina, além de outros procedimentos cirúrgicos no útero. 
Para um diagnóstico adequado deve-se preconizar uma ultrassonografia com cuidado em pacientes de alto risco, sendo que um diagnóstico precoce permite uma revisão adequada dos procedimentos, avaliação dos riscos bem como os benefícios a ser instituídos em cada caso.

A histerectomia é a opção que leva a menor morbidade materna, e esta não deve ser protelada se o tratamento conservador se mostrar ineficaz frente ao quadro materno comprometido por sinais de choque, infecções ou demais complicações que apresentem risco iminente à vida.

Entretanto a conduta tomada durante o transoperatório evidenciou de forma unânime a preservação do útero, demostrando que a equipe pensou de forma equilibrada e conjunta mantendo a visão holística do conceito de saúde biopsicossocial ao ponderar a idade da paciente, o insucesso das gravidezes passada, o desejo manifestado em tornar-se mãe pela mesma e o suporte disponível para o manejo e tratamento adequado pós cirúrgico.

\section{Referências}

ARTICO, L. G. et al. Alterações histopatológicas em placentas humanas relacionadas às síndromes hipertensivas. Revista Brasileira de Ginecologia e Obstetrícia, Rio de Janeiro, v. 31, n. 1, p. 1016, jan., 2009. Disponível em: <http://www.scielo.br/scielo.php?script=sci_arttext\&pid=S0100$72032009000100003 \& \operatorname{lng}=$ en\&nrm=iso $>$. Acesso em: 12 jan. 2018.

HUBINONT, C. et al. A clinic-pathologic study of placenta percreta. International Journal of Gynecology \& Obstetrics, v. 140, n. 3, p. 365-369, jan., 2018. Disponível em: <https://www.ncbi.nlm.nih.gov/pubmed/29194617>. Acesso em: 12 jan. 2018.

JAUNIAUX, E.; BHIDE, A. Prenatal ultrasound diagnosis and outcome of placenta previa accreta after caesarean delivery: A systematic review and meta-analysis. American Journal of Obstetrics \& Gynecology, v. 217, n. 1, p. 27-36, jul., 2017. Disponível em: <https://www.ncbi.nlm.nih.gov/pubmed/28268196>. Acesso em: 19 dez. 2017.

JAUNIAUX, E.; COLLINS, S. L.; BURTON, G. J. The placenta accreta spectrum: Pathophysiology and evidence-based anatomy for prenatal ultrasound imaging. American Journal of Obstetrics \& Gynecology, v. 218, n. 1, p. 75-87, jan., 2017. Disponível em: <https://www.ncbi.nlm.nih.gov/pubmed/28599899>. Acesso em: 19 dez. 2017.

LEVIN, G. et al. An unusual case of placenta percreta in a patient with myotonic dystrophy. European Journal of Obstetrics and Gynecology and Reproductive Biology, v. 221, dez., 2017. 
Disponível em: <http://www.ejog.org/article/S0301-2115(17)30602-4/fulltext>. Acesso em: 10 jan. 2018.

MATSUZAKI, S. et al. Successful anticoagulant therapy for disseminated intravascular coagulation during conservative management of placenta percreta: a case report and literature review. BMC Pregnancy Childbirth, v. 17, p. 443, 2017. Disponível em: <https://www.ncbi.nlm.nih.gov/pmc/articles/PMC5747084/>. Acesso em: 19 dez. 2017.

NARASIMHA, A.; VASUDEVA, D. S. Spectrum of changes in placenta in toxemia of pregnancy. Indian Journal of Pathology and Microbiology, v. 54, n. 1, p. 15-20, jan., 2011. Disponível em: <https://www.ncbi.nlm.nih.gov/pubmed/21393870?dopt=Abstract>. Acesso em: 12 jan. 2017.

ROLNÁ, M.; MATLÁK, P.; DVOŘÁČKOVÁ, J. Placenta percreta as a cause of massive intraabdominal bleeding. Ceska Gynekologie, v. 82, n. 6, p. 478-481, 2017. Disponível em: <https://www.ncbi.nlm.nih.gov/pubmed/29302982>. Acesso em: 10 jan. 2018.

SUN, W. et al. Safety and efficacy of preoperative abdominal Aortic balloon occlusion in placenta increta and/or percreta. Journal of Surgical Research, v. 222, p. 75-84, nov., 2017. Disponível em: <http://www.journalofsurgicalresearch.com/article/S0022-4804(17)30649-2/fulltext>. Acesso em: 19 dez. 2017.

TANTBIROJN, P.; CRUM, C. P.; PARAST, M. M. Pathophysiology of Placenta Creta: The Role of Decidua and Extravillous Trophoblast. Placenta, v.29, n. 7, p. 639-645, jul., 2008. Disponível em: < http://www.placentajournal.org/article/S0143-4004(08)00145-8/fulltext>. Acesso: 19 dez. 2017. 